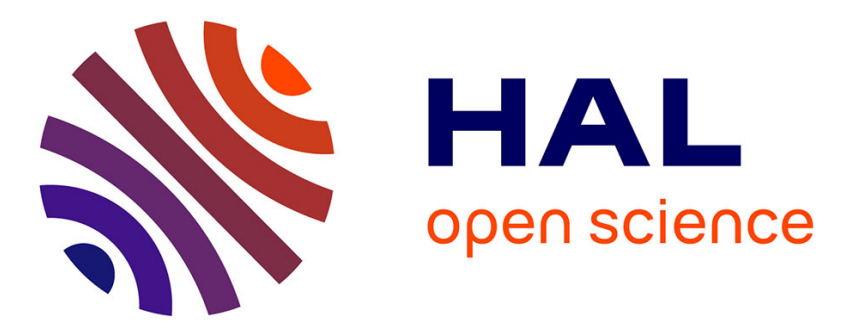

\title{
Motion-compensated optical coherence tomography based on higher-order regression for real-time volumetric imaging
}

\author{
Ruizhi Zuo, Kristina Irsch, Jin Kang
}

\section{- To cite this version:}

Ruizhi Zuo, Kristina Irsch, Jin Kang. Motion-compensated optical coherence tomography based on higher-order regression for real-time volumetric imaging. Proc. SPIE 11630, Optical Coherence Tomography and Coherence Domain Optical Methods in Biomedicine XXV, Mar 2021, Online Only, United States. pp.116301N, 10.1117/12.2577906 . hal-03447694

\author{
HAL Id: hal-03447694 \\ https://hal.science/hal-03447694
}

Submitted on 24 Nov 2021

HAL is a multi-disciplinary open access archive for the deposit and dissemination of scientific research documents, whether they are published or not. The documents may come from teaching and research institutions in France or abroad, or from public or private research centers.
L'archive ouverte pluridisciplinaire HAL, est destinée au dépôt et à la diffusion de documents scientifiques de niveau recherche, publiés ou non, émanant des établissements d'enseignement et de recherche français ou étrangers, des laboratoires publics ou privés. 


\section{Motion-compensated optical coherence tomography based on higher-order regression for real-time volumetric imaging}

Zuo, Ruizhi, Irsch, Kristina, Kang, Jin

Ruizhi Zuo, Kristina Irsch, Jin U. Kang, "Motion-compensated optical coherence tomography based on higher-order regression for real-time volumetric imaging," Proc. SPIE 11630, Optical Coherence Tomography and Coherence Domain Optical Methods in Biomedicine XXV, 116301N (5 March 2021); doi: $10.1117 / 12.2577906$

SPIE. Event: SPIE BiOS, 2021, Online Only 


\title{
Motion-compensated Optical Coherence Tomography based on higher-order regression for real-time volumetric imaging
}

\author{
Ruizhi Zuo ${ }^{\mathrm{a}}$, Kristina Irsch ${ }^{\mathrm{b}, \mathrm{c}}$, Jin U. Kang ${ }^{\mathrm{a}, \mathrm{c}}$ \\ a Johns Hopkins University, Whiting School of Engineering, Baltimore, MD, USA; ${ }^{\mathrm{b}} \mathrm{CNRS}$, \\ Vision Institute, Paris, France; ' Johns Hopkins University, School of Medicine, Baltimore, MD, \\ USA
}

Optical Coherence Tomography (OCT) has evolved into a powerful clinical tool, with a wide range of applications in ophthalmology. However, most OCT systems for real-time volumetric (3D) and in vivo imaging suffer from image distortion due to motion artifacts induced by involuntary and physiological movements of the living tissue. Several methods have been proposed to obtain motion-free images, yet they are generally limited in their applicability due to long acquisition times, requiring multiple volumes [1], and/or the need for additional hardware [2]. Here we propose and analyze a motion-compensated 3D-OCT imaging system that uses a higher-order regression analysis and show that it can effectively correct the motion artifacts within 0 to $5 \mathrm{~Hz}$ in real time without requiring additional hardware.

\section{Method}

The scanning protocol of the proposed motion-compensated 3D-OCT system is shown in Figure 1. Three reference images in the slow-axis are utilized to obtain reference images within the C-Scan volume and compensate for the motion artifacts. Note that mainly axial motion is considered in this analysis, such as the one induced by involuntary vascular pulsation and respiration. We define $d$ as the distance between adjacent references. $L$ is the width of the B-scan raw image. $\alpha$ is defined as the ratio between $d$ and $L$. The system is implemented in an in-house built $100 \mathrm{kHz}$ swept-source OCT centered at $1060 \mathrm{~nm}$ with $110 \mathrm{~nm}$ tuning range.

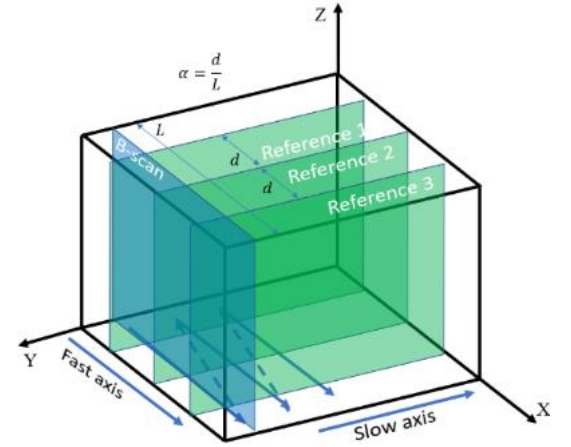

Figure 1. Schematic of the scanning protocol of the motion-compensated 3D-OCT system.

The flowchart of the system algorithm is shown in Figure 2. The algorithm consists of two main steps, namely preprocessing and motion compensation. First, we apply a gaussian filter to every B-Scan of the raw C-scan data and remove the saturation artifacts introduced by light reflected from a highly specular surface generating signals that are over the dynamic range of the data-acquisition system. We simplified the saturation removal method based on minimum variance mean-line subtraction (MVMS) $[3,4]$, to reduce the computation cost. Specifically, the A-line value of position $(\mathrm{x}, \mathrm{y})$ will be assigned as subtraction of the mean value of raw data $A_{\Omega}(x, y)$ from $A_{\Omega}(x, y)$. After thresholding and the connect component analysis, we remove any noisy portion, including tissue inside the cornea, then generate the initial segmentation. We revise the surface using the pixel with the largest gradient around the initial segmentation and fit it with a polynomial curve. To test this preprocessing algorithm, we applied it to clinical OCT images of corneas with normal and abnormal surface topography (e.g. from bullous keratopathy and keratoconus). As shown in Figure 3 , the results indicate that the 4 th order polynomial is accurate enough to represent the topological surface of both the healthy and

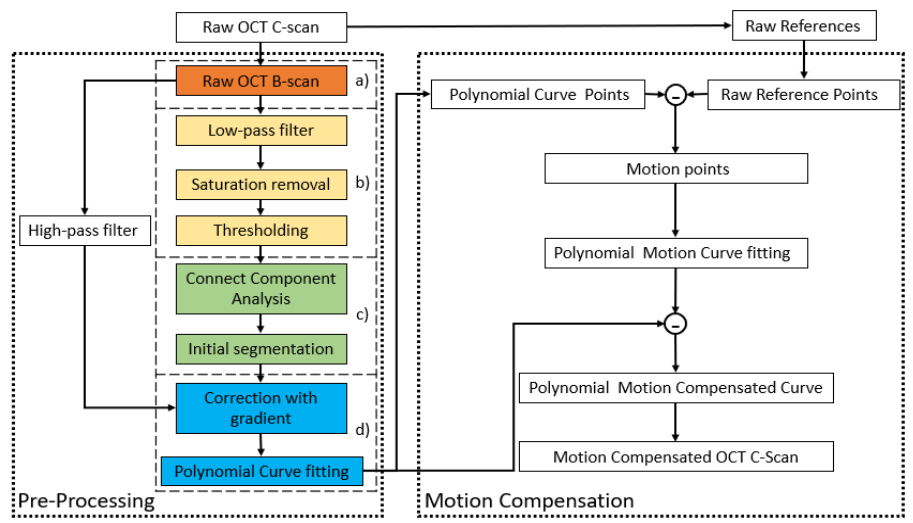

Figure 2 Flowchart of system algorithm, with (left) pre-processing (a through d) and (right) motion-compensation steps.

pathological corneas. 

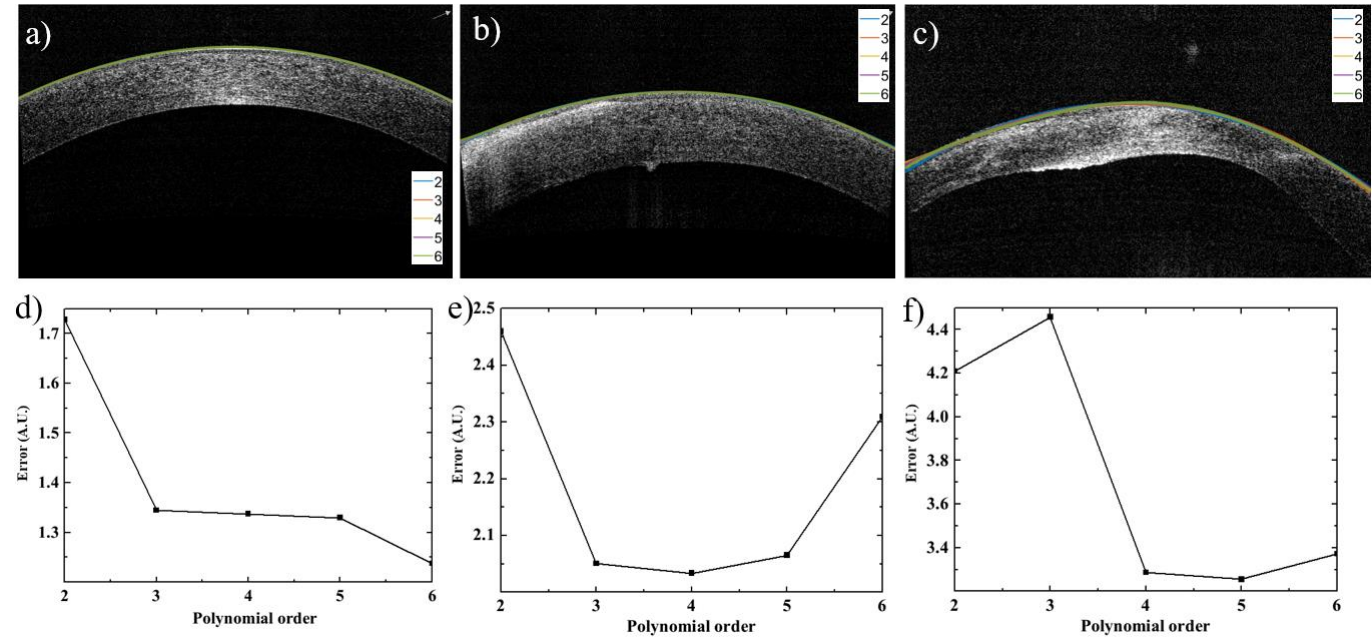

Figure 3. Polynomial fitting results of corneal boundary. a), b), and c) shows the different polynomial order fitting results and corresponding fitting error d), e) and f) of the normal cornea, cornea with bullous keratopathy, and cornea with keratoconus, respectively.

We compare the B-scan surface detection result with the three reference images and obtain the motion during the three intersecting planes. Then, the movement of every A-scan within the B-scan is predicted and compensated by the polynomial fitting.

\section{Results}

Because most involuntary motion frequency of the human body ranges from 0 to $5 \mathrm{~Hz}$ [5], we explored the movement over this frequency range in this study. Figure 4. a)-c) shows an example of the motion-compensation result during corneal C-scan volume imaging with axial movement around $1 \mathrm{~Hz}$. As another example, Figure 4. d)-f) shows volumetric imaging of a phantom with motion around $3.6 \mathrm{~Hz}$. The algorithm successfully corrects the motion artifacts in these two frequency settings.
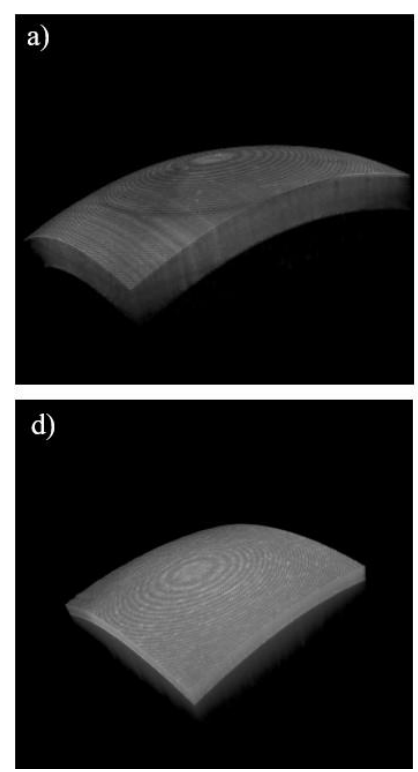

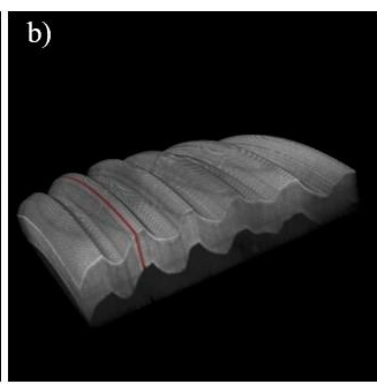

e)

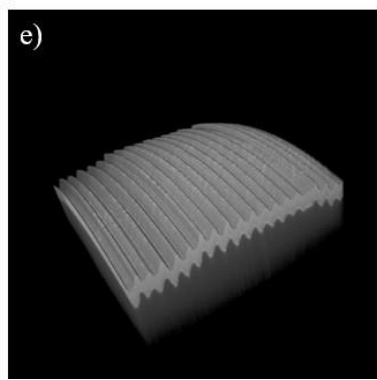

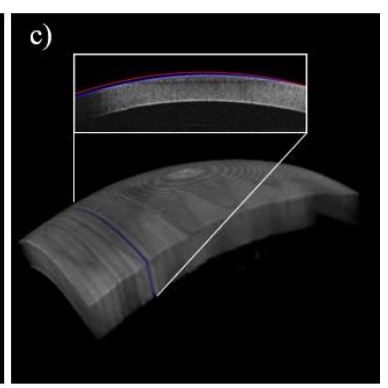

f)

Figure 4. Motion-compensation result of (top) cornea and (bottom) phantom C-scan volume. a) is the static reference of the cornea, b) is the cornea with motion around $1 \mathrm{~Hz} . \mathrm{c}$ ) is the reconstructed corneal image after motion compensation. The red line in b) and blue line in c) represents the B-scan location, representing the corneal surface before and after compensation, respectively. Both are also shown in the inset of c), which provides a direct comparison between pre- and post-compensation. d), e) and f) are the static reference of the phantom, phantom with motion around $3.6 \mathrm{~Hz}$, and reconstruction of phantom after motion compensation. 
The Root Mean Square Error (RMSE) is used as our quantitative metric of the result, to measure the difference between the reference and compensated sample in micrometers. To test the performance of the system and the algorithm, we compensated for the motion around $1 \mathrm{~Hz}$ using different order polynomial fittings with four cornea samples as shown in Figure 5. The result shows that 4th order fitting achieves the best fitting accuracy, with minimal RMSE.

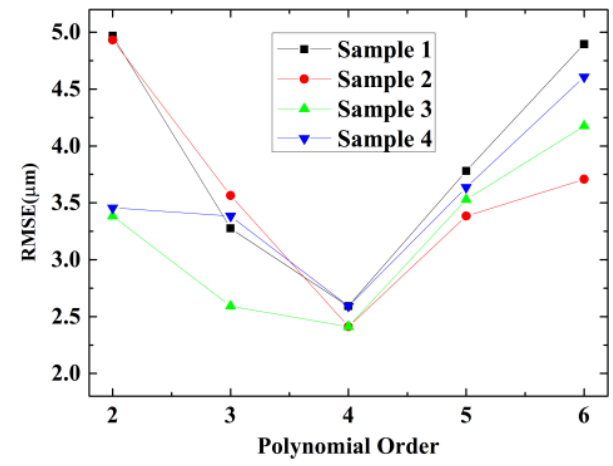

Figure 5. RMSE between four compensated cornea samples and the static reference with different polynomial orders.

The compensation result could be affected by the distance between the adjacent reference planes, which is defined as $\alpha$. Since the middle reference is fixed in the center of the $\mathrm{x}$-direction, $\alpha$ can range from 0 to 0.5 . Figure 6 shows that $\alpha$ around 0.37 achieves the best result, that is, a minimal RMSE, below around $2.6 \mu \mathrm{m}$.
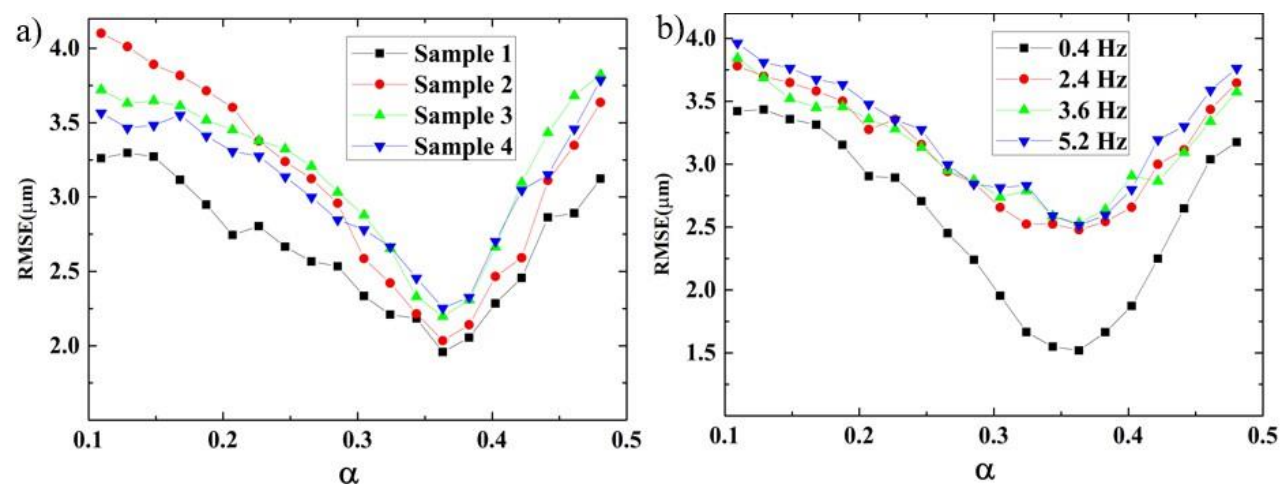

Figure 6. Error between motion compensation and static reference as a function of $\alpha$ for 4 different a) corneas with $1 \mathrm{~Hz}$ axial motion and $\mathrm{b}$ ) phantoms with diverse motion frequencies.

In conclusion, we propose and analyze a motion-compensated 3D-OCT system and show that it can compensate motion over the frequency range from 0 to $5 \mathrm{~Hz}$ effectively with less than $2.6 \mu \mathrm{m}$ error. Implementing such motion compensation into clinical OCT systems has the potential to improve the reliability of 3D imaging and reduce associated computational cost.

\section{References}

[1] Lezama, J., Mukherjee, D., McNabb, R. P., Sapiro, G., Kuo, A. N., and Farsiu, S. "Segmentation guided registration of wide field-of-view retinal optical coherence tomography volumes," Biomedical Optics Express, 7(12), 4827 (2016).

[2] LaRocca, F., Nankivil, D., Farsiu, S., and Izatt, J. A. "Handheld simultaneous scanning laser ophthalmoscopy and optical coherence tomography system," Biomedical Optics Express, 4(11), 2307-2321 (2013).

[3] Moon, S., Lee, S. W., and Chen, Z. "Reference spectrum extraction and fixed-pattern noise removal in optical coherence tomography," Optics Express, 18(24), 24395-24404 (2010).

[4] Huang, Y., and Kang, J. U., "Real-time reference A-line subtraction and saturation artifact removal using graphics processing unit for high-frame-rate Fourier-domain optical coherence tomography video imaging," Optical Engineering, 51(7), 073203 (2012).

[5] McNabb, R. P., LaRocca, F., Farsiu, S., Kuo, A. N., and Izatt, J. A. "Distributed scanning volumetric SDOCT for motion corrected corneal biometry," Biomedical Optics Express, 3(9), 2050-2065 (2012). 